\title{
TU/e EmonONEN

\section{The interplay of air and sand: Faraday heaping unravelled}

\section{Citation for published version (APA):}

Gerner, van, H. J., Hoef, van der, M. A., Meer, van der, D., \& Weele, van der, J. P. (2007). The interplay of air and sand: Faraday heaping unravelled. Physical Review E - Statistical, Nonlinear, and Soft Matter Physics, 76(5), 051305-1/7. [051305]. https://doi.org/10.1103/PhysRevE.76.051305

DOI:

10.1103/PhysRevE.76.051305

Document status and date:

Published: 01/01/2007

\section{Document Version:}

Publisher's PDF, also known as Version of Record (includes final page, issue and volume numbers)

\section{Please check the document version of this publication:}

- A submitted manuscript is the version of the article upon submission and before peer-review. There can be important differences between the submitted version and the official published version of record. People interested in the research are advised to contact the author for the final version of the publication, or visit the $\mathrm{DOI}$ to the publisher's website.

- The final author version and the galley proof are versions of the publication after peer review.

- The final published version features the final layout of the paper including the volume, issue and page numbers.

Link to publication

\section{General rights}

Copyright and moral rights for the publications made accessible in the public portal are retained by the authors and/or other copyright owners and it is a condition of accessing publications that users recognise and abide by the legal requirements associated with these rights.

- Users may download and print one copy of any publication from the public portal for the purpose of private study or research.

- You may not further distribute the material or use it for any profit-making activity or commercial gain

- You may freely distribute the URL identifying the publication in the public portal.

If the publication is distributed under the terms of Article 25fa of the Dutch Copyright Act, indicated by the "Taverne" license above, please follow below link for the End User Agreement:

www.tue.nl/taverne

Take down policy

If you believe that this document breaches copyright please contact us at:

openaccess@tue.nl

providing details and we will investigate your claim. 


\title{
Interplay of air and sand: Faraday heaping unravelled
}

\author{
Henk Jan van Gerner, ${ }^{1}$ Martin A. van der Hoef, ${ }^{1}$ Devaraj van der Meer, ${ }^{1}$ and Ko van der Weele ${ }^{2}$ \\ ${ }^{1}$ Faculty of Science and Technology, University of Twente, P.O. Box 217, 7500 AE Enschede, The Netherlands \\ ${ }^{2}$ Department of Mathematics, University of Patras, 26500 Patras, Greece
}

(Received 21 June 2006; revised manuscript received 12 September 2007; published 26 November 2007)

\begin{abstract}
We report on numerical simulations of a vibrated granular bed including the effect of the ambient air, generating the famous Faraday heaps known from experiment. A detailed analysis of the forces shows that the heaps are formed and stabilized by the airflow through the bed while the gap between bed and vibrating bottom is growing, confirming the pressure gradient mechanism found experimentally by Thomas and Squires [Phys. Rev. Lett. 81, 574 (1998)], with the addition that the airflow is partly generated by isobars running parallel to the surface of the granular bed. Importantly, the simulations also explain the heaping instability of the initially flat surface and the experimentally observed coarsening of a number of small heaps into a larger one.
\end{abstract}

DOI: 10.1103/PhysRevE.76.051305

PACS number(s): 45.70. $-\mathrm{n}, 05.65 .+\mathrm{b}, 47.11 .-\mathrm{j}$

\section{INTRODUCTION}

Faraday heaping is one of the most celebrated and beautiful examples of the effect of air on granular matter. When a box with fine dry sand is vertically vibrated or tapped, its initially flat surface turns into a landscape of small heaps, which in the course of time tend to coarsen into larger heaps [1-5]. At very low pressure, when the air drag on the particles can be ignored, no heaping of any kind is observed, implying that the ambient air plays a crucial part in the heaping instability, as was already noted by Faraday [6]. The subject has received considerable attention since its discovery by Chladni and Faraday, especially in recent years with the increased interest in granular matter. These studies-all experimental - have led to several explanations as to why the heaps keep a stable shape, including internal avalanches [1], horizontal pressure gradients [3], and the stability of inclined surfaces [7]. In this paper we present numerical simulations of the Faraday heaping effect, including the coarsening process of the small heaps into larger heaps. Although numerical simulations of tilting in a water-immersed granular bed have been reported earlier [8], this is the first time, to our knowledge, that a genuine Faraday heap is simulated. The simulations allow us to obtain a detailed insight into the physical mechanism at work, elucidating the role of the ambient air, and discriminating between the previously proposed explanations of the heaping effect.

\section{NUMERICAL MODEL}

For the simulation we developed a hybrid granular dynamics (GD) computational fluid dynamics (CFD) code $[9,10]$. The GD code calculates the particle trajectories from Newton's law, with the particle-particle interactions being given by a three-dimensional (3D) soft sphere collision model including tangential friction. The CFD code evaluates the full Navier-Stokes equations by a finite difference method. For the two-way coupling between the particles and the gas phase, the drag force relations described in Ref. [11] are used [12]. The simulated system, Fig. 1, contains 13500 spherical particles $\left(\rho=2500 \mathrm{~kg} / \mathrm{m}^{3}\right)$ of average diameter $0.5 \mathrm{~mm}$, with a Gaussian size distribution $(\sigma=0.055 \mathrm{~mm})$ to avoid excessive ordering of the bed. In all simulations the coefficient of restitution is set to 0.90 for the normal direction, and to 0.33 for the tangential direction. For the particlewall interaction the same collision parameters are used as for the particle-particle interaction. The friction coefficient is set to 0.2. All these parameters are typical for glass spheres in a glass box. The dimensions of the box are $100 \times 50$ $\times 2.1 \mathrm{~mm}^{3}(W \times H \times D)$, i.e., four particles fit in the depth direction and the mean height of the granular bed in rest is about fourteen particle diameters. The box is closed airtight, containing air at atmospheric pressure without gas leaving or entering the system. For the numerical simulations, the box is divided in $80 \times 60 \times 1(W \times H \times D)$ CFD cells. It was found that using more CFD cells did not produce different results.

\section{STEADY STATE HEAP}

Starting with a flat bed, and vertically vibrating it using a sinusoidal driving with frequency $f=6.25 \mathrm{~Hz}$ and amplitude
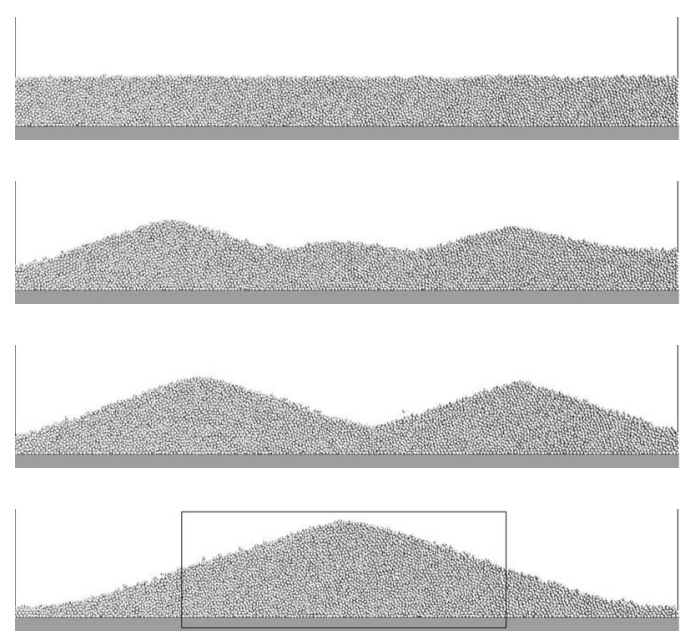

FIG. 1. Numerically simulated Faraday heaping in a vibrated granular bed after $0,30,55$, and 240 driving cycles. After a few cycles, some slight surface ripples start to grow into small heaps, which coarsen into larger heaps until a steady state with a single Faraday heap is reached. 


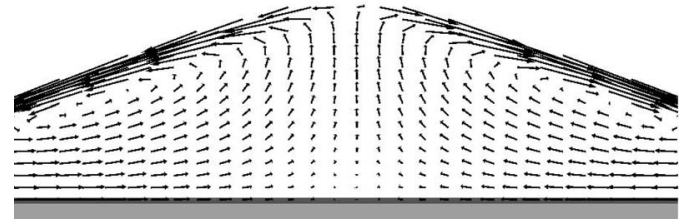

FIG. 2. Circulation in the Faraday heap: The arrows indicate the particle displacement (multiplied by 2 for clarity) during one driving cycle, averaged over 25 cycles. The part of the heap shown here corresponds to the black box in Fig. 1.

$a=10 \mathrm{~mm}$ [corresponding to a dimensionless acceleration $\left.\Gamma=a(2 \pi f)^{2} / g=1.6\right]$, we typically observe the following series of events (Fig. 1): Already after a few cycles, some random surface fluctuations are seen to grow into small heaps. After this, on a much longer time scale, the small heaps merge together into larger heaps until finally a steady state with a single heap is reached. Under vacuum conditions (i.e., if we turn off the gas-particle interaction in the simulations) no heap is formed, in accordance with the experimental findings [2,6]. Also, if we remove the air once a heap is formed, it disappears within 15 cycles.

We first focus on the steady state with one heap and address the question how the heap keeps its stable shape. A good starting point is given by Fig. 2, which shows the dynamical equilibrium that is present within the heap. The particles are seen to be in constant motion, following a circulation pattern that consists of two convection rolls, in which the outward avalanche in the upper surface layers is balanced by a collective inward motion of the particles in the interior of the heap. This pattern confirms the circulation proposed by Faraday [6] and found experimentally by Behringer $e t a l$. [4]; half of the pattern (one convection roll) was found numerically for water-immersed beds by Milburn et al. [8].

How is the circulation sustained? To reveal and understand the mechanism, we follow the heap during a complete vibration cycle, see the snapshots at phase angles $90^{\circ}, 171^{\circ}$, $234^{\circ}$, and $360^{\circ}$ in Fig. 3. The vertical bands of gray marker particles illustrate again the circulation of Fig. 2, the black arrows indicate the drag force of the air on the particles. Initially, the bottom plate and bed are moving upwards together. The first snapshot in Fig. 3 is taken at $90^{\circ}$, when the bed has already detached from the bottom and a gap has started to grow between bottom and bed, causing a region of low pressure underneath the heap. The isobars are indicated with black lines in this figure. The pressure above the heap is approximately constant, because the air resistance in the heap is much higher than above the heap. For this reason, the isobars run parallel to the surface of the heap, resulting in an airflow perpendicular to this surface. Furthermore, the pressure is lowest underneath the center of the heap because the air enters more easily through the sides than through the center. This generates a downward airflow through the heap (strong at the sides where the heap is thin, weaker in the center), with a noticeable inward component. The air drag thus accelerates the particles down and inwards.

At $171^{\circ}$, the heap is in free flight. The contact forces between the particles as well as the air drag are very small at

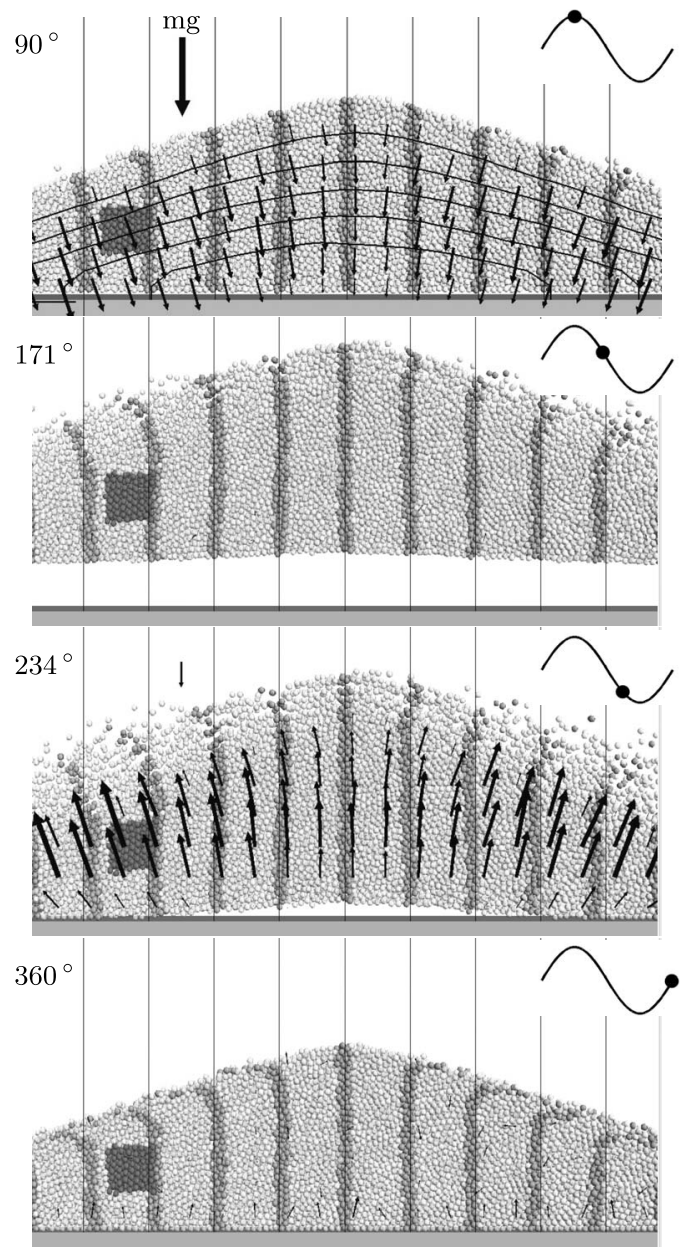

FIG. 3. The stable Faraday heap (see Fig. 2) at four successive phases during one driving cycle. The arrows indicate the air drag force on the particles averaged per CFD cell (one out of every four arrows is shown); at $234^{\circ}$, when the drag force is very strong, the arrows are scaled by a factor of $1 / 3$ compared to those at the other phase angles. For comparison, the gravitational force on a particle is shown in the $90^{\circ}$ figure (and also at $234^{\circ}$ ); the ratio of gravity and air drag forces, which is comparable to the Bagnold number [14], is of order one in this simulation. The dynamics of the rectangular ensemble of dark particles is further analyzed in Fig. 4. A video of one driving cycle can be found here [13].

this stage, so the particles within the heap maintain their inward motion and hence the number of particles near the center increases. It can be seen that the bed itself becomes slightly curved, because the downward drag at $90^{\circ}$ was larger at the sides.

At $234^{\circ}$, the bed is coming down again, and the sides have in fact already reached the bottom. The particles now experience a large upward and outward drag force due to the increased air pressure between the box and the descending granular bed. However, the collision with the bottom will rapidly compactify the heap, so that the particles get locked and cannot move anymore.

Finally, at $360^{\circ}$ the heap is in rest except for a thin layer of particles that avalanches down its slopes. Since we are in a steady state, this precisely neutralizes the surplus of particles at the heap's center built up during the previous stages, 


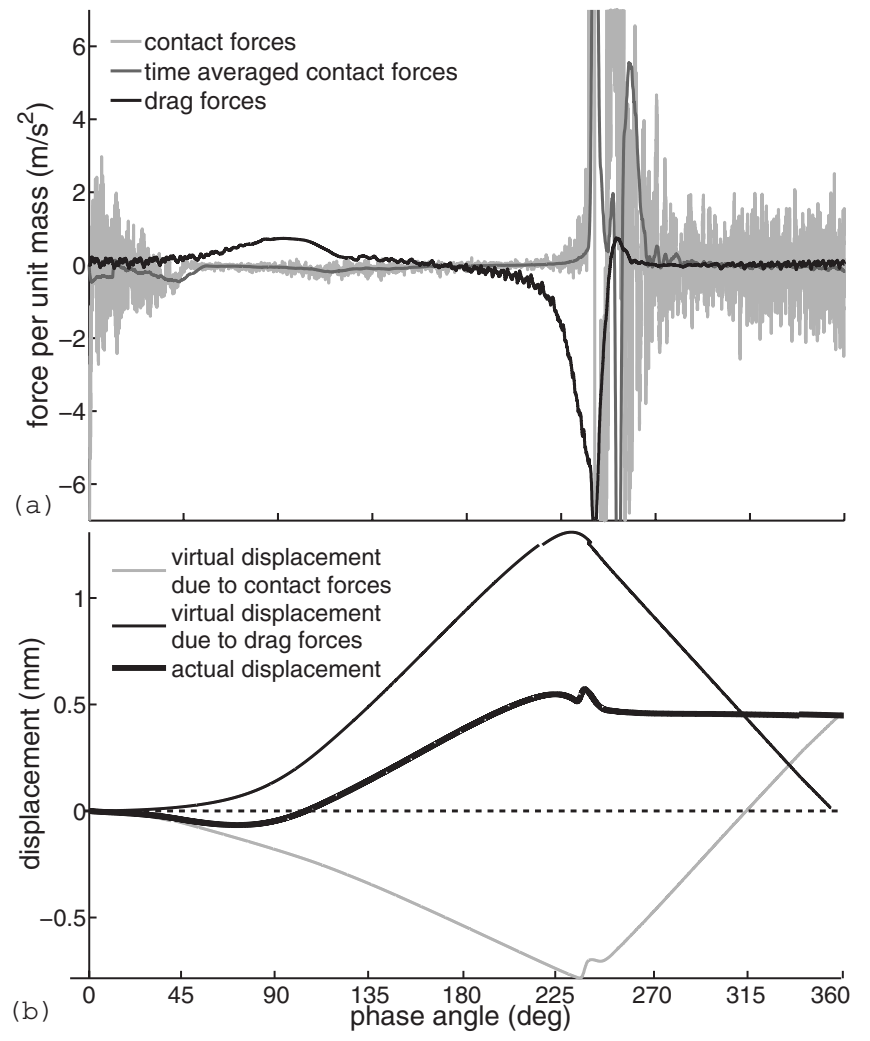

FIG. 4. (a) The averaged horizontal components of the contact and drag forces (per unit mass) on the rectangular ensemble of dark particles in Fig. 3, as a function of time during a complete driving cycle. Positive forces point towards the center of heap. (b) The virtual displacements due to the contact and drag forces individually, and their sum, i.e., the actual horizontal displacement of the ensemble. The net displacement during one cycle $(0.43 \mathrm{~mm}$ towards the center of the heap) is already reached at $260^{\circ}$. After that, the heap is fully compactified and the particles in the ensemble are locked until a new cycle starts.

and thereby completes the circulation pattern of Fig. 2 .

The above findings are similar to the experimental observations of Thomas and Squires [3], who found-from floor pressure measurements at multiple locations-a horizontal component of the pressure gradient which drives the particles inward during the first part of the cycle, when the particles are free to move. However, the horizontal component of the pressure gradient not only arises from air entering the heap more easily through the sides (resulting in a horizontal pressure gradient at the floor) but also from the isobars running parallel to the surface [15], which induces a horizontal component of the pressure gradient in the bed. We further investigated the significance of this second mechanism by carrying out a simulation in which the top of the box is closed and the bottom is permeable for air (i.e., a constant pressure at the bottom is prescribed): Also in this case heaps are formed, solely due to the fact that the isobars are parallel to the surface, without any pressure gradient at the bottom. The inward pressure gradient due to the isobars running parallel to the surface plays an especially important role at onset of heaping, when the horizontal pressure gradient on the floor is relatively small and the main contribution to the hori-

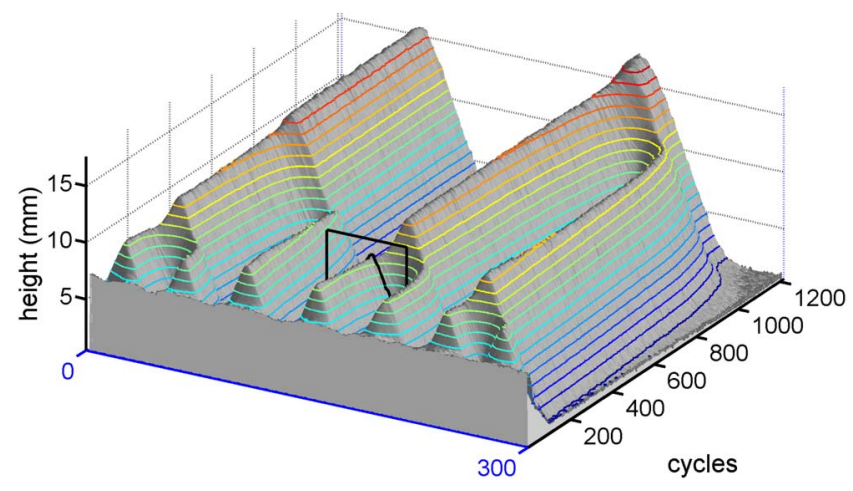

FIG. 5. (Color online) Evolution of the heap pattern in a box three times as wide as that in Fig. 1. A video of this evolution can be found here [13].

zontal component of the pressure gradient stems from the curved isobars along the surface.

For a quantitative description of the heaping mechanism we show in Fig. 4(a) the horizontal components of the contact and drag forces per unit mass on the rectangular ensemble in Fig. 3, during one driving cycle. In Fig. 4(b) we integrate these forces twice, which yields the virtual displacement of the ensemble due to the contact and drag forces individually. The sum of these two virtual displacements is the actual horizontal displacement of the ensemble, given by the solid black curve in Fig. 4(b).

During the very first stages, the particles are seen to move slightly outward due to the contact forces, which are initially quite large, but rapidly decrease as the bed loosens up. At $40^{\circ}$ they become practically zero. At this point the gap between the bed and the bottom starts to grow, inducing the inward air drag discussed above, which reaches a maximum value around $90^{\circ}$. This drag force succeeds in turning the motion of the ensemble around at $75^{\circ}$, and the particles start to move inward. This motion is maintained up to $220^{\circ}$. At $200^{\circ}$ the gap between bottom and bed is largest and the ensemble hardly experiences any forces. After $200^{\circ}$ the gap becomes smaller again and the air drag is now directed in the opposite direction, reversing the motion of the ensemble at $220^{\circ}$. Shortly afterwards $\left(\approx 240^{\circ}\right)$, the heap collides with the bottom-sides first—and this sends a shock wave through the bed with a large horizontal inward component. As a result, the motion is turned around again towards the center of the heap [exemplified by the small hump in the total displacement curve, Fig. 4(b)] and the bed compactifies, causing the wild turmoil of contact forces in Fig. 4(a) at $240^{\circ}-260^{\circ}$ and fixating the particles. Figure 4(b) shows that around $260^{\circ}$ the ensemble comes to a standstill, its final horizontal displacement after one cycle being $0.43 \mathrm{~mm}$ inward, slightly less than a particle diameter.

\section{EVOLUTION OF AN INITIALLY FLAT BED}

So far we focused on the steady state of a single Faraday heap, but how does this steady state come into existence? Figure 5(a) shows the evolution of an initially flat bed (which was created by giving all particles a random velocity 

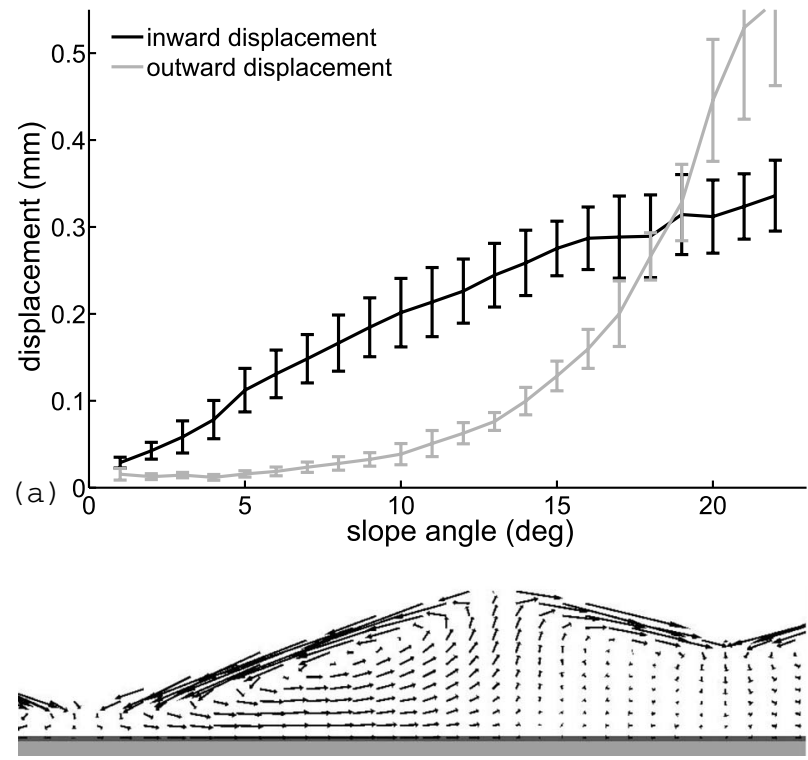

(b)

FIG. 6. (a) Averaged horizontal particle displacement in a heap, due to the inward air drag (black) and outward avalanching (gray), as a function of the growing slope angle, during the first stage when the heaps are formed. The displacements balance each other at a slope of $18.5^{\circ}$. (b) Particle displacement during one cycle in the heap indicated by the frame in Fig. 5, which is about to merge with the neighboring heap to the right. The displacements are averaged over five cycles around 240 cycles and multiplied by two for clarity.

with a standard deviation of $0.1 \mathrm{~m} / \mathrm{s}$ ) as a function of the number of cycles. The width of the box $W$ is here increased from 0.1 to $0.3 \mathrm{~m}$ (to accommodate a reasonable number of heaps) and 40500 instead of 13500 particles are used, thus keeping the mean depth of the granular bed constant. We observe a two-stage coarsening process $[16,17]$ : (a) A fast initial stage where seven small heaps are formed within 50 cycles, followed by (b) a slow second stage where the heaps merge and combine into two larger heaps; it is anticipated that these heaps will eventually merge into one single heap, as in Fig. 1, however, since the merging slows down considerably with a decreasing number of heaps, the required computing time is beyond our computer resources. Interestingly, during the whole coarsening process the slopes of the heaps have a nearly constant angle of $18.5^{\circ}$, which is lower than the static angle of repose $\left(22.7^{\circ}\right.$, determined by an independent simulation).

With respect to stage (a), detailed simulations show that as soon as a slight ripple appears on the surface, the isobars follow the surface and the local air drag directly acquires a noticeable horizontal component, dragging particles towards the center via the mechanism of Fig. 3. Initially, the slope of the ripple is so small that there is hardly any avalanching. Thus it steadily grows, increasing its slope and thereby the number of particles that avalanche down, until at some specific angle (here $18.5^{\circ}$ ) an equilibrium is reached between the fluxes due to avalanching and inward drag force. This is illustrated in Fig. 6(a), which shows the averaged horizontal displacement per cycle of inward and outward moving particles separately, as a function of the angle of the slope. The

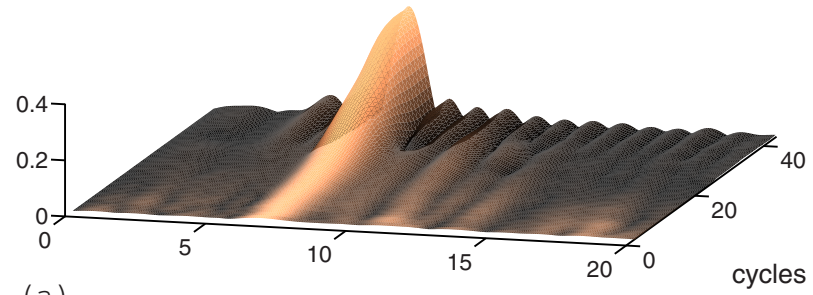

(a)

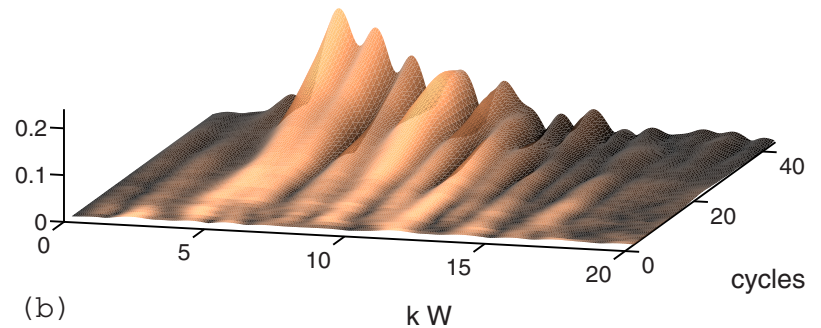

FIG. 7. (Color online) (a) Fourier transform of the first 45 cycles of Fig. 5 as a function of the wave number $k$ (made dimensionless by the width of the box $W$ ). The color indicates the relative value of the Fourier transform per cycle, the height is the absolute value. The initial number of seven heaps is already present in the initial condition and grows stronger as the heaps grow during the first 30 cycles. (b) Fourier transform of the first 45 cycles of a simulation with different initial conditions, resulting in a landscape of heaps without any specific wavelength.

measurements for heap angles up to $18.5^{\circ}$ are obtained during the first 50 cycles and averaged over the five center heaps in Fig. 5. The measurements for heap angles between $18.5^{\circ}$ and $22^{\circ}$ are obtained by pouring particles on the seven heaps after 50 cycles, until the static angle of repose is reached, and then restart the vibration of the system. The error bars denote the standard deviation of the data.

After stage (a), seven heaps are present in the box with a width $(W)$ of $0.3 \mathrm{~m}$, independent of the vibration parameters: Simulations carried out at different frequencies and amplitudes always result in this wave number. An almost constant wave number was also found experimentally in Ref. [18], although the wavelength found there is around $6 \mathrm{~mm}$, while the wavelength in our simulations is around $43 \mathrm{~mm}$. A Fourier transform of Fig. 5 shows that the wave number is already strongly present in the initial condition [Fig. 7(a)], although the difference in height between the peaks and valleys is less than one particle diameter. The question arises if the wave number of seven is inherent to the heaping instability, or just a result of the initial condition of the granular bed. To answer this question, the initial granular bed is first vibrated for $5 \mathrm{~s}$ at a frequency of $200 \mathrm{~Hz}$ and an amplitude of $1 \times 10^{-5} \mathrm{~m}$, resulting in a granular bed with a nearly flat surface. The difference in height between the peaks and valleys is less than one particle diameter and a Fourier transform of the surface shows small humps at many wave numbers. Subsequently, a heaping simulation with this bed as initial condition is carried out, resulting after 45 cycles in a collection of heaps with different sizes and no specific wave number [Fig. 7(b)], although wave numbers above 15 are not present anymore; a detailed analysis shows that the heaplets with larger wave numbers merge into heaps with small wave numbers before they reach their steady state angle. It may be 
concluded that the wave number during stage (a) is very sensitive to the initial condition and that the wave number of 7 is not related to the vibration parameters or particle and box dimensions.

To explain the physics of stage (b), i.e., why the heaps merge, we proceed analogously as for the single Faraday heap and consider the averaged displacement of particles during one cycle in the region indicated with black lines in Fig. 5 after 240 cycles [Fig. 6(b)]. The arrows show that the inward motion inside the left part of the selected heap is much larger than in the right part. This asymmetry in the displacement field is caused by the fact that the granular bed is thinner at the left side of the heap, so the inward drag force is stronger here. This will result in a displacement of the entire heap towards the right, and the merging with the neighboring heap. Thus the coarsening of heaps takes place primarily because heaps move towards each other and not because the height of one heap steadily increases while the height of another heap decreases, as suggested in Ref. [19].

\section{INFLUENCE OF DIFFERENT PARAMETERS}

\section{Air drag, friction, and restitution coefficient}

The outcome of a heaping simulation depends on a number of parameters. In order to obtain insight in the influence of these parameters, simulations are carried out where one parameter is slightly changed after each vibration cycle. The rate of change of a parameter was chosen such that decreasing the rate of change any further did not produce significantly different results. The initial condition for each simulation is the steady state heap shown in Fig. 1 and is indicated by the markers in Fig. 8 .

One of the most important parameters which influences the heaping is the ratio of the air drag and the gravity force, comparable to the (reciprocal) Bagnold number that plays an important role in the description of aerial transport of sand in deserts [14]. If it is reduced to zero, no heaping of any kind is observed.

The drag force acting on the granular bed can be represented as the drag force on a single isolated particle in the limit of zero Reynolds number (the Stokes-Einstein relation) multiplied by a dimensionless empirical function $f$ that depends on the packing fraction $\phi$ and Reynolds number Re, e.g., the Ergun relation [20] or the drag force relation described in Ref. [11]: $F_{\mathrm{drag}}=3 \pi \mu d U f(\phi, \mathrm{Re})$, where $\mu$ is the viscosity of the medium, $d$ the diameter of the particles, and $U$ the velocity of the air relative to the bed.

The gravitational force on each particle (of mass $m$ ) is simply $F_{g}=m g=\rho \pi g d^{3} / 6$, with $\rho$ the density of the particles and $g$ the gravitational acceleration. Hence the dimensionless number that gives the ratio of the (typical) drag force and gravity takes the form

$$
N=\frac{F_{\mathrm{drag}}}{F_{g}}=\frac{18 \mu U}{\rho g d^{2}} f(\phi, \mathrm{Re}) .
$$

In this equation, the particle size and density ( $d$ and $\rho$ ), gravity $(g)$, and viscosity $(\mu)$ are the control parameters, whereas $\phi, \operatorname{Re}$, and $U$ are response parameters of the system, which
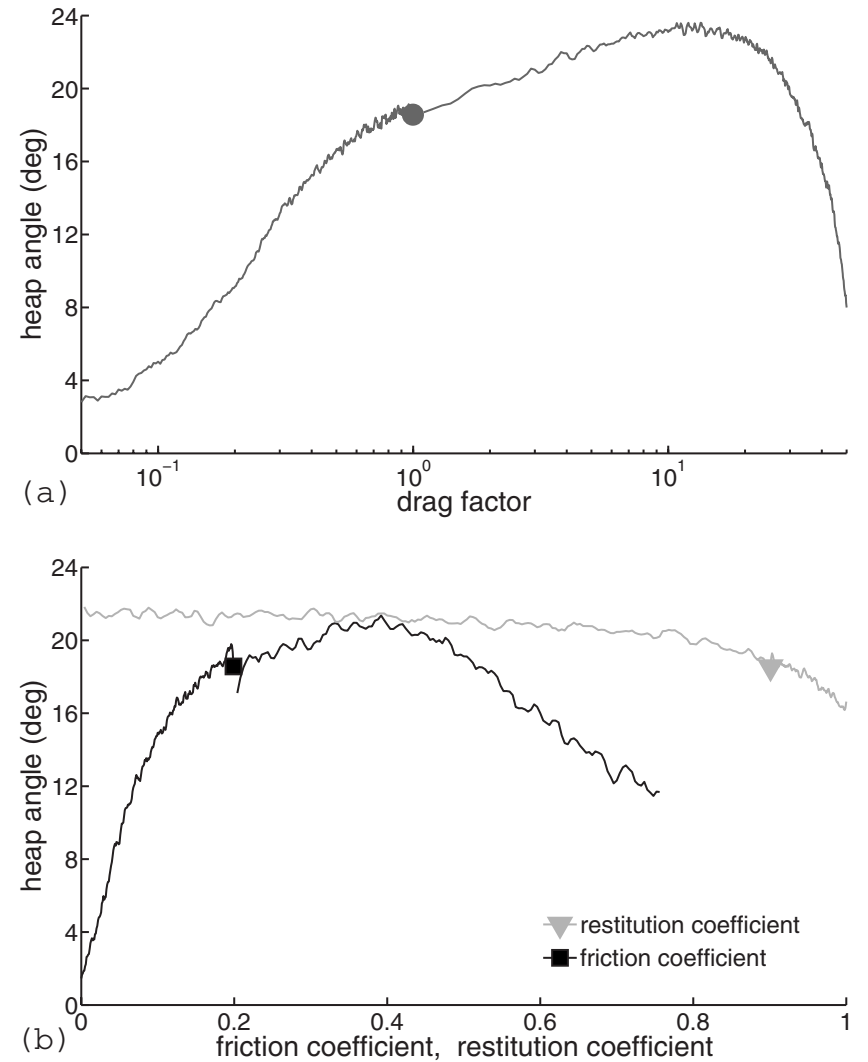

FIG. 8. (a) Heap angle as a function of the air drag factor. (b) Heap angle as a function of restitution and friction coefficient. The measurements are obtained from simulations in which one parameter is changed slightly after each vibration cycle. The initial condition for each simulation, corresponding to the steady state heap of Fig. 1, is indicated with a marker.

vary through the system and change if we vary any one of the control parameters. This means that it is not feasible to systematically vary the dimensionless number [Eq. (1)] via one of the physical control parameters. Moreover, a modification of the control parameters may not only change the ratio $N$, but also other quantities that influence the heaping. Therefore, we choose to modify the level of the air drag $F_{\text {drag }}$ artificially by introducing an air drag factor, i.e., a factor by which we multiply the air drag obtained from the drag relation. The result is shown in Fig. 8(a): If the air drag factor is decreased from its default value 1 towards zero, the heap angle becomes smaller and smaller, just as expected. Likewise, if we increase the air drag factor, the heap angle grows and reaches a maximum at a drag factor of 20. Increasing the drag factor still further results in a decrease of the heap angle; detailed simulations show that for a drag factor of 40 the air drag acting on the particles becomes so strong that the bed does not detach from the plate anymore and hence the particles can hardly move inward.

For comparison we also performed a limited set of simulations in which we varied a physical control parameter (particle size and density, gravity, viscosity). We found that changing either the viscosity $\mu$ or the particle density $\rho$ gave similar results as a corresponding change of the air drag factor [21]. However, decreasing the particle diameter $d$ by a 
factor of 7 turned out to give a quite different result than simply multiplying the drag factor by 49 ; in particular, in the former case the granular bed is much more diluted (i.e., $\phi$ becomes smaller) during the free flight phase. Finally, changing the gravitational acceleration $g$ leads to different results (than the corresponding reciprocal change in the air drag factor) because it not only influences the dimensionless ratio $N$, but the dimensionless acceleration $\Gamma$ as well [22]. Therefore, it can be concluded that the influence of the particle density $\rho$ and the viscosity $\mu$ on the heap formation is controlled exclusively by the ratio $N$, which is not true for the diameter $d$ or gravitational acceleration $g$.

Changing the friction coefficient has a pronounced influence on the heap angle. Because the angle of repose of a pile of particles is $0^{\circ}$ without any friction, the heap must disappear when the friction coefficient is reduced to zero. On the other hand, increasing the friction above 0.4 also results in a decrease of the heap angle. This can also be explained since the inward motion of the particles due to the air drag is hindered by the increased particle-wall and particle-particle friction.

Interestingly, the restitution coefficient only has a small influence on the heap angle at these vibration parameters. Even at a restitution coefficient of 1 (the elastic case), the energy dissipation due to friction is sufficient to prevent the heap from collapsing during impact. However, simulations show that the restitution coefficient can have a marked influence at more vigorous shaking conditions: When the box is vibrated with a dimensionless acceleration $\Gamma$ of 2.4 (at a frequency $f=12.5 \mathrm{~Hz}$ ), the heap collapses with a restitution coefficient of 0.9 but remains stable with a restitution coefficient of 0.5 .

\section{Vibration amplitude and acceleration}

The vibration amplitude $a$ and dimensionless acceleration $\Gamma$ are important parameters for Faraday heaping. The influence of these parameters is systematically studied in simulations at five different frequencies $(4.17,5,6.25,9$, and $17 \mathrm{~Hz}$ ). In each simulation, the dimensionless acceleration $\Gamma$ starts with 1.2 and is increased (via the amplitude $a$ ) with 0.005 after each vibration cycle. This value was chosen such that decreasing this value any further did not produce significantly different results. The initial condition for the simulations is the steady state heap in Fig. 1. The heap angle as a function of vibration amplitude $a$ and dimensionless acceleration $\Gamma$ that results from these simulations, is indicated by colors in the straight lines in Fig. 9(a). The heap angle slowly decreases with increasing acceleration until a dimensionless acceleration of approximately 1.9, above which the heap collapses and the heap angle reduces to $0^{\circ}$.

Simulations show that the inward motion during one vibration cycle (and therefore the velocity of the Faraday circulation) depends on both vibration acceleration and amplitude. The straight lines in Fig. 9(b) show that the inward motion during one vibration cycle, is small for low vibration amplitudes, that is, at equal values of $\Gamma$ the magnitude of the inward motion grows for increasing $a$ [23]. This is reflected in the time required to evolve from an initially flat surface to

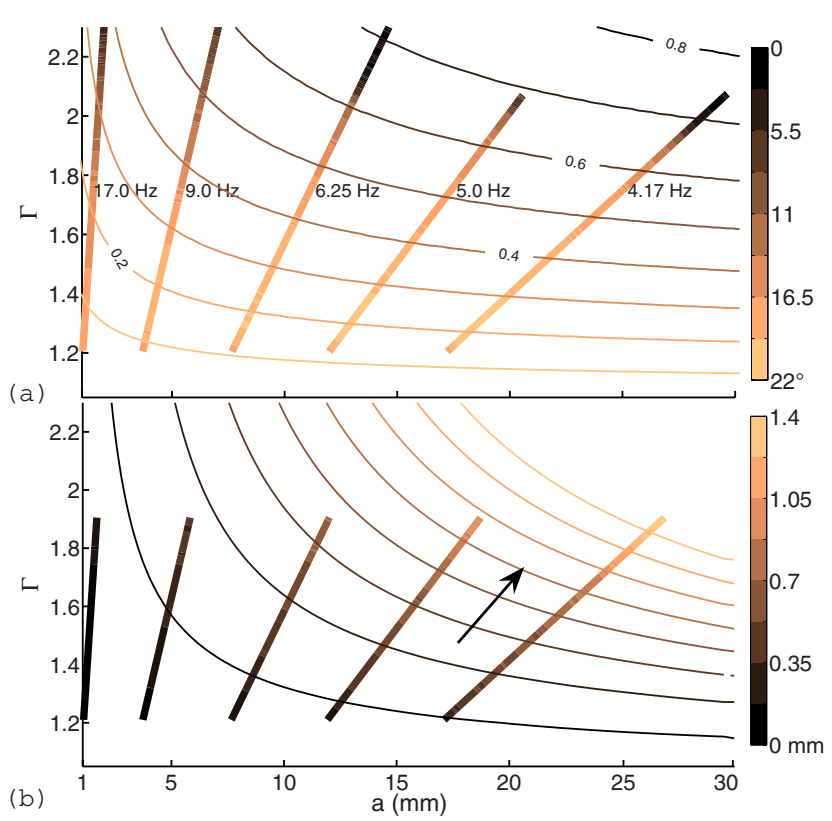

FIG. 9. (Color online) (a) Straight lines: Heap angle as a function of vibration amplitude $a$ and dimensionless acceleration $\Gamma$. The color bar indicates the heap angle in degrees. The measurements are obtained from simulations at five different frequencies as indicated. Contour lines: Impact velocity obtained from the model. The increment between the contour lines is $0.1 \mathrm{~m} / \mathrm{s}$. (b) Straight lines: Inward particle motion obtained from the same simulations. The color bar indicates the inward displacement per particle per vibration cycle in $\mathrm{mm}$. The inward displacement increases in the direction of the arrow. Contour lines: Inward motion of the particles obtained from the model. The maximum value of the inward motion in the model is scaled to match the maximum value in the simulation.

seven small heaps: Around 20 seconds in a simulation with a vibration amplitude $a=2.5 \mathrm{~mm}$ (and frequency $f=12.5 \mathrm{~Hz}$ ) and just $3 \mathrm{~s}$ in a simulation with $a=22 \mathrm{~mm}$ (and $f$ $=4.17 \mathrm{~Hz}) ; \Gamma$ is 1.6 in both simulations.

The simulations show that the heap disappears with increasing acceleration, despite the fact that the inward motion increases. In order to gain more insight in the influence of the vibration amplitude and acceleration, a simple onedimensional model was created which consists of a completely inelastic object (representing the total granular mass) bouncing on a vibrating plate. It is assumed that the velocity of the air is equal to the velocity of the vibrating plate. The vertical component of the air drag on the object is calculated with the Ergun drag force relation [20] on a single particle, where it is assumed that the porosity of the granular mass has a constant value of 0.5 . Because the pressure contours are parallel to the heap surface (and the air drag force is perpendicular to the pressure contours), the horizontal drag force on the particles is calculated by multiplying the vertical drag force with the sine of a steady heap angle of $18.5^{\circ}$. Integrating the horizontal drag force twice (until impact of the object with the plate) results in the inward motion of the particles during one vibration cycle, as indicated by the contour lines in Fig. 9(b). The results from the model correspond well with the results from the simulations: The inward motion (and therefore the Faraday circulation) increases with increasing amplitude and acceleration. 
The model also calculates the impact velocity of the object on the vibrating plate. This impact velocity is indicated by contour lines in Fig. 9(a). In order to determine the relation between the impact velocity and the outward displacement of the particles due to avalanching, we carried out simulations in which a heap was thrown with different velocities onto a horizontal plate. The simulations showed that avalanching strongly increases with increasing impact velocity; the precise relation, however, depends on various parameters as heap size, porosity before impact, etc.

So why does the heap disappear with increasing acceleration while the inward motion of the particles increases? From the model and the simulations, it follows that the inward motion due to air drag increases with vibration amplitude and acceleration, but a limit is reached when the impact of the heap on the plate becomes so severe that the outward motion due to avalanching can never be compensated by the inward motion of the particles.

\section{CONCLUSION}

In conclusion, the simulations give a detailed insight into the mechanism of Faraday heaping, elucidating in particular the role of the ambient air. Faraday's original assertion that the air must play a key role has been fully verified [6]; the experiments by Thomas and Squires [3] have been validated; their proposed pressure-gradient mechanism has been identified as the correct one; moreover, we have shown the important role being played by the isobars running parallel to the surface of the granular bed.

The simulations also explain how the system evolves from an initially flat surface to a single stable heap: very small initial surface deflections are unstable, leading to a transient state of several small heaps, which (on a much longer time scale) move toward each other due to the reduced inward drag force on the side of a heap that is close to another one.

Finally, we have shown that the Faraday circulation velocity increases with vibration amplitude and acceleration. A limit is reached when the impact of the bed on the plate becomes too strong and the outward motion due to avalanching becomes larger than the inward motion due to the air drag. That is why the heaping phenomenon breaks down at large shaking strengths.

\section{ACKNOWLEDGMENTS}

We want to thank Christiaan Zeilstra for his support with the computer code, and Hans Kuipers and Detlef Lohse for many inspiring and insightful discussions. This work is part of the research program of the Stichting FOM, which is financially supported by NWO.
[1] C. Laroche, S. Douady, and S. Fauve, J. Phys. (France) 50, 699 (1989).

[2] H. K. Pak, E. van Doorn, and R. P. Behringer, Phys. Rev. Lett. 74, 4643 (1995).

[3] B. Thomas and A. M. Squires, Phys. Rev. Lett. 81, 574 (1998).

[4] R. P. Behringer, E. van Doorn, R. R. Hartley, and H. K. Pak, Granular Matter 4, 9 (2002).

[5] H. M. Jaeger, S. R. Nagel, and R. P. Behringer, Rev. Mod. Phys. 68, 1259 (1996).

[6] M. Faraday, Philos. Trans. R. Soc. London 52, 299 (1831).

[7] J. Duran, C. R. Phys. 3, 217 (2002).

[8] R. J. Milburn, M. A. Naylor, A. J. Smith, M. C. Leaper, K. Good, M. R. Swift, and P. J. King, Phys. Rev. E 71, 011308 (2005).

[9] M. A. van der Hoef, M. Ye, M. van Sint Annaland, A. T. Andrews IV, S. Sundaresan, and J. A. M. Kuipers, Adv. Chem. Eng. 31, 65 (2006).

[10] N. G. Deen, M. van Sint Annaland, M. A. van der Hoef, and J. A. M. Kuipers, Chem. Eng. Sci. 62, 28 (2007)

[11] M. van der Hoef, R. Beetstra, and J. A. M. Kuipers, J. Fluid Mech. 528, 233 (2005).

[12] Our simulations show that the precise form of the drag force is not very critical: Simulation carried out with the Ergun drag force relation [20] led to similar results.

[13] See EPAPS Document No. E-PLEEE8-76-017711 for a video of the Faraday heap during one driving cycle and for a video of the evolution of the heap pattern. For more information on EPAPS, see http://www.aip.org/pubservs/epaps.html

[14] J. Duran, Sand, Powders, and Grains. An Introduction to the
Physics of Granular Materials (Springer, New York, 2000).

[15] Thomas and Squires use a linear interpolation between floor pressures and the constant surface pressure to obtain a pressure within the bed. This interpolation results in isobars that follow the surface-this is, however, not mentioned in their article [3], nor is the important consequence of the resulting inward pressure gradient near the surface.

[16] M. C. Cross and P. C. Hohenberg, Rev. Mod. Phys. 65, 851 (1993).

[17] D. van der Meer, J. P. van der Weele, and D. Lohse, J. Stat. Mech.: Theory Exp. (2004) P04004.

[18] E. van Doorn and R. P. Behringer, Phys. Lett. A 235, 469 (1997).

[19] J. Duran, Phys. Rev. Lett. 87, 254301 (2001).

[20] S. Ergun, Chem. Process Eng. (London) 48, 89 (1952).

[21] Even if the viscosity of the fluid is reduced to zero, a small drag force acts on the particles, because part of the drag force does not depend on the viscosity, but on the pressure drop over a particle (the so-called form drag, which is included in the empirical models). However, for realistic parameters, the form drag is much smaller than the viscous drag and changing $\mu$ leads to similar results as changing the air drag factor.

[22] The dimensionless acceleration $\Gamma$ is important because, in a vacuum, it is the only parameter that determines the phase angle at which the granular mass detaches from and collides with the bottom of the box during a vibration cycle.

[23] The simulation results of the inward motion above $\Gamma \approx 1.9$ are very scattered (because the heap does not remain in the same position) and are therefore not shown in the plot. 